\title{
Combination of Time and Spatial Filtering to Improve Echo Reduction in Antenna Measurements
}

\author{
Manuel Sierra-Castañer, Javier García-Gasco Trujillo, \\ Pilar González-Blanco, Manuel José López Morales
}

Francesco Saccardi, Lars J. Foged

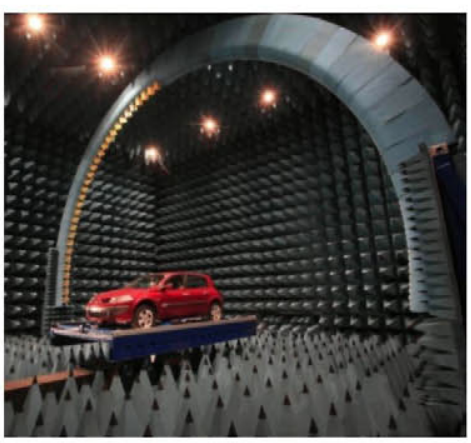

Fig. 1. Measurement of VHFantennas on a vehicle in Renault facilities

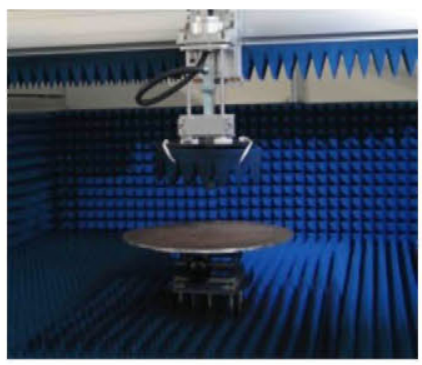

Fig. 2. Measurement a Radial Line Slot Antenna in Tokyo Tech Facilities.

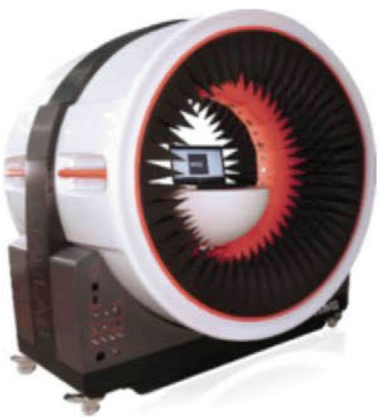

Fig. 3. MVG StarLab@ semi-open multiproble measurement system.

Fig. 1 to Fig. 3 show three applications: the first case shows the measurement of an anechoic chamber for antennas on vehicles measurements. In this case, the minimum frequency 
can be $30 \mathrm{MHz}$, where the absorbing material does not work properly. The application of echo cancelling techniques becomes necessary for having satisfactory results. The second case is the measurement of Radial Line Slot Antennas in a Planar Scanner. In this case, the interaction between AUT and antenna probe is significant, and the application of these methods improve drastically the quality of the results. The third case (Fig. 3) shows the multiprobe system StarLab ${ }^{\circ}$. This system can be moved in the laboratory easily, but on the other hand absorbing material only covers the area in front of the AUT. The application of these post processing tools can improve the quality of the measuement of non directive antennas.

The main objectives of this paper are first the analysis of the advantages and drawbacks of each method; and second, how to combine different methods in order to have more accurate results.

The paper is divided in the following sections. Section 2 details briefly the theory of the different methods. Section 3 establishes the main advantages and disadvantages of each method in a general way. Section 4 presents the results on the application of different methods to the measurements of a dipole on multiprobe antenna measurement system, summarizing and explaining the reasons on the improvement of one or another method. Finally, Section 5 concludes the paper, explaining how to combine different methods for improving he results.

\section{OVERVIEW OF THE DifFERENT AlgORITHMS}

This section presents a brief overview of the different families of methods. Although there are many other methods, and they can be divided in different ways, we divided the methods in three families: spatial filtering, modal filtering and time filtering. For each family, there are also plenty of implementations. The authors do not pretend to do a wide review of all of them, but to try to extract the main advantages and drawbacks of the methods and analyze the usability of them for the measurement of a non-directive antenna.

\section{A. Spatial filtering}

Spatial filtering methods are called to those ones where the near of far field pattern is transformed to the domain of the antenna under test through a source reconstruction technique. There are different ways of getting this: in [1] UPM researchers applied a FFT to the Plane Wave Spectrum in order to transform from $\mathrm{k}_{\mathrm{x}} / \mathrm{k}_{\mathrm{y}}$ domain to $\mathrm{x} / \mathrm{y}$ domain in the proximity of the AUT. If the reconstruction is done on a surface much larger than the AUT, the echoes can be detected and filtered out. The main advantage of the method is the speed (since it uses interpolations, FFT and plane wave propagation). The main disadvantage is that the reconstructed field is limited to a plane, due to the fact of using the Fourier Transform. Also, if the source of reflection is not well established, and the surface where the sources are calculated is not large enough, the FFT produces aliasing and the echoes are not suppressed. Fig. 4 shows the application of this technique to a RADAR antenna in a cylindrical outdoor range (ITM-CEAR cylindrical near field system in Guadalajara-Spain).
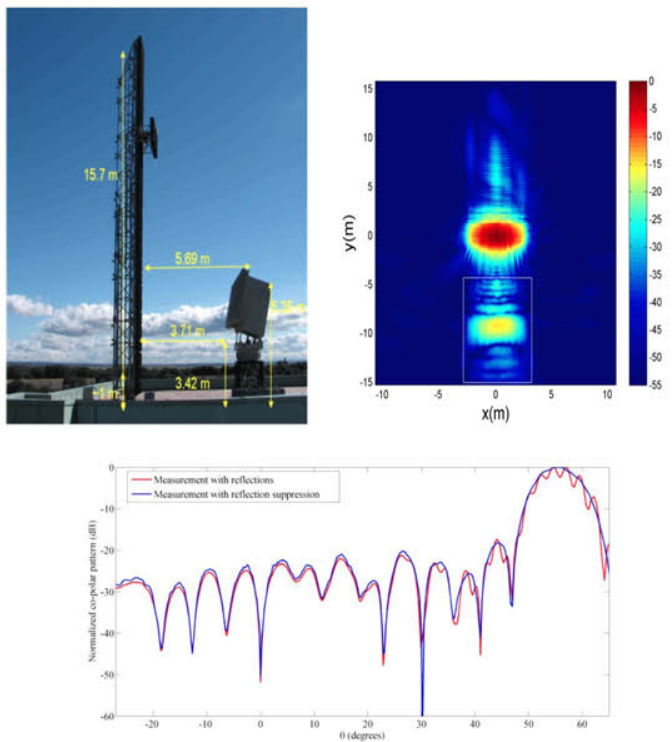

Fig. 4. Application of Holographic Technique to a RADAR L-band Antenna.

The field is reconstructed on a large surface, and the sources coming from the AUT can be separated from the sources coming from the reflection in the ground (right part of the figure). In the lower figure, it is observed the improvement in the result of the radiation pattern.

However this can be solved by using other electromagnetic methods solving directly the integral equation that relates the sources and the fields. MVG and Politecnico di Torino researchers investigated this method in [2]. Also, this technique is commercially available in two different Software packages (DIATOOL $^{\mathrm{C}}$ from TICRA and INSIGHT ${ }^{\mathrm{C}}$ from MVG). The main advantage of the method is that the result is not limited to a plane. Any volume covering the AUT can be used for the source reconstruction. Another advantage is that the user does not need to extend the surface of the source reconstruction to be able to cancel the reflections. Obviously, the main drawback is the time and memory required for the processing.

\section{B. Modal Filtering}

We call modal filtering to the transformation from near field pattern to spherical or cylindrical waves and the filtering of the field in that domain. In fact, the pattern can also be transformed to plane wave spectrum, but in this case, the process is the one of the spatial filtering. Also, this method could be included in the family of spatial filtering, since there is a relation between the number of modes and the size (and position in the antenna measurement system) of AUT.

There are three main Software packages for this function: MARS $^{\mathcal{C}}$ from NSI is based on the decomposition of the far field or any of the near field configurations (planar, cylindrical or spherical) in cylindrical modes. The modes are filtered out depending on the dimensions of the AUT. This method is explained in [3]. ISOFILTER ${ }^{\mathrm{C}}$ from MI-Technology uses spherical modes decomposition of the near field. In the new domain, the field can be filtered out since there is a relation between the antenna size, position in the measurement system and modes where the field is contained. This method is widely explained in [4]. 

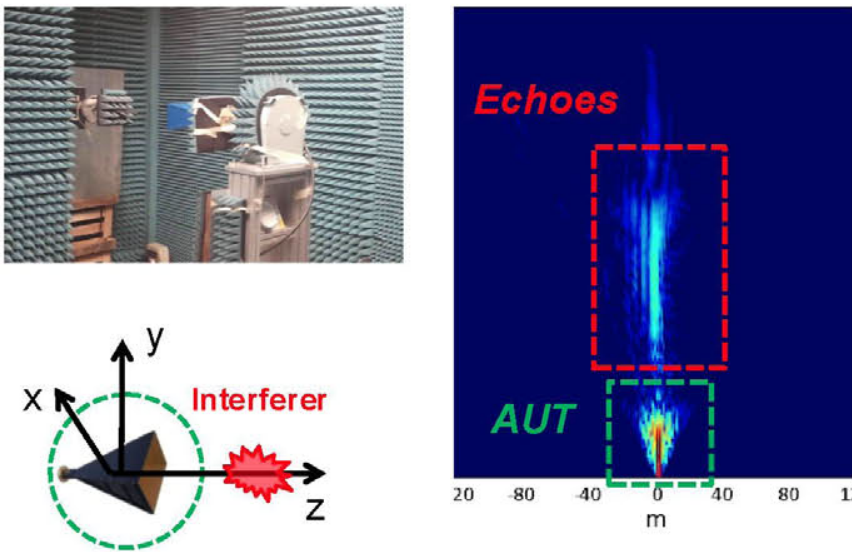

Fig. 5. Application of MV-Echo to a horn antenna measurement in a echo environment.

Also, spherical mode decomposition is used by MVG in the Software package MV-Echo [5]. Again, the same principle is used: transformation from near field to spherical wave domain, displacement of the field and filtering out of the information out of the valid modes. Fig. 5 shows the application of MVEcho to the measurement of a horn antenna placed in a spherical measurement system where a metallic plate has been placed. The decomposition in spherical modes shows that the information of the AUT can be separated from the information of the echoes.

\section{Time Filtering}

Time filtering was the first method used for echo reduction. The first approach is the use of a FFT to transform from the frequency domain to the time domain (Fig. 6). The process is very simple: the samples measured at different frequencies are transformed to time domain (via inverse FFT), in that domain the echoes and the direct ray can be filtered and a new FFT is applied to reconstruct the field. This technique is applied to near field antenna measurement in [6]. The main drawback is the number of samples and the required frequency band to have enough resolution in time domain.

Also, Matrix Pencil Method can be used for the same purpose [7]. The main advantage of this method is that the samples in frequency do not need to be equally spaced. Therefore, the method can be applied in a general way. Also, more sophisticated applications of Matrix Pencil give directly the user the exponentials where there is information.

Similar to the application of the Matrix Pencil Method is the application of a non-uniform DFT in the solution of the frequency to time-domain transformation. The authors implemented this last method for the results of Section IV.

\section{AdVANTAGES AND DRAWBACKS OF EACH METHOD}

This section compares the properties of three methods belonging to each one of the families: INSIGHT ${ }^{\circ}, \mathrm{MV}^{-E_{c h o}}{ }^{\circ}$, and Time domain filtering applying Non uniform DFT. These same methods will be used for the measurement of the dipole antenna.

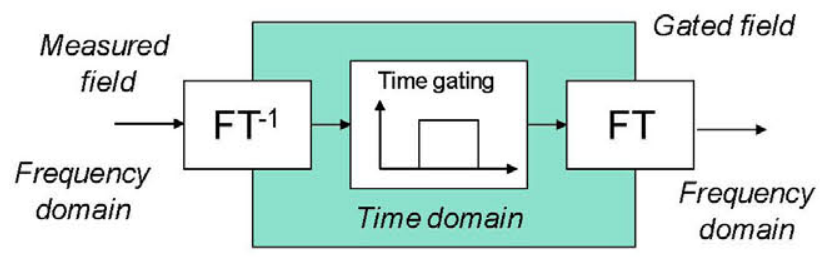

Fig. 6. Echo cancellation using time gating technique.

\begin{tabular}{|r|c|c|c|c|}
\hline Algorithm & $\begin{array}{c}\text { Processing } \\
\text { Time }\end{array}$ & $\begin{array}{c}\text { Need of } \\
\text { Multi } \\
\text { Frequency }\end{array}$ & $\begin{array}{c}\text { Need of } \\
\text { Geometry } \\
\text { Information }\end{array}$ & $\begin{array}{c}\text { Availability } \\
\text { in Spherical } \\
\text { Scanner }\end{array}$ \\
\hline Ansight & Large & NO & YES & \\
\hline MV Echo & Low & NO & Dimension & Partial \\
\hline Mn Flter & Low & YES & Not much & YES \\
\hline
\end{tabular}

Fig. 7. Advantages and Drawbacks of the methods.

Fig. 7 shows the main advantages of each method. For time domain, the main problem is the requirement of multifrequency measurements. This makes the method not valid for some applications. However, it could be good for planar measurements, where multifrequency is usually employed. Also, it is valid for multiprobe measurement systems where the acquisition time is not so critical. With respect the processing time, source reconstruction requires much more time than the others, due to use of integral equation method. With respect the need of information of the geometry, time domain filtering requires less information than the others. With respect the validity of the method for all kind of measurement set-ups, the more flexible is time domain, since the processing is done point to point. In the other cases, when the AUT is not fixed, the signal from the echoes is not perfectly cancelled. That means that the two first methods work very well for planar scanners, well for cylindrical and multiprobe systems, and have some problems for classical spherical systems.

\section{APPLICATION OF THE METHODS}

The previous three methods have been applied to the measurement of the dipole antenna SD1900, working at $1900 \mathrm{MHz}$. The dipole has been measured in a StarLab system (Fig. 8) where plate has been placed in front of the AUT. The dipole has been chosen due to the omni-directional radiation pattern, a worst case for the analysis of reflections. In this case, the StarLab is placed in an anechoic environment. The plate is a square of side dimension of $334 \mathrm{~cm}$, placed in front of the dipole, orthogonal to the arch. This is the worst position for using the MV-Echo or the Insight. However, the results will be good enough for both algorithms. Time filtering has been used from measurements in the frequency band $1.7 \mathrm{GHz}$ to $2.2 \mathrm{GHz}$ with a step of $10 \mathrm{MHz}$. Spatial and modal filtering have been applied to the central frequency measurements $(1.9 \mathrm{GHz})$.

Fig. 9 shows the results for the vertical pattern of the dipole. The reference is considered the raw data including modal filtering. It is observed that Spatial Filtering (INSIGHT) 


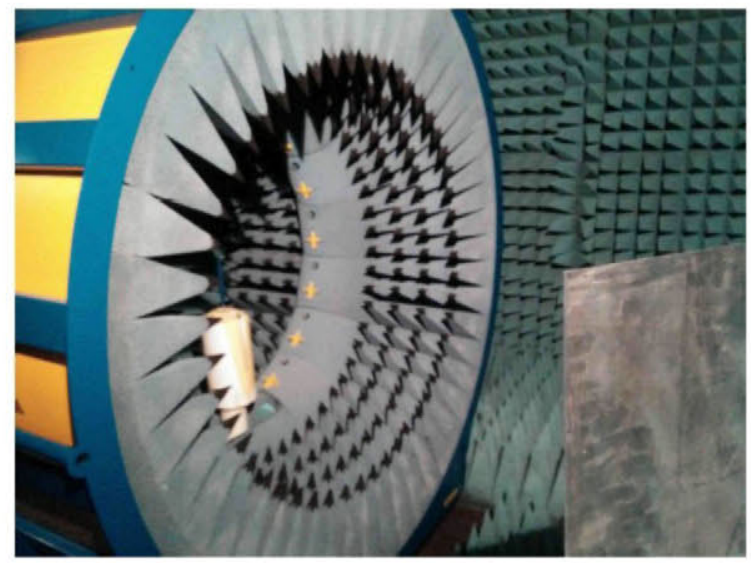

Fig. 8. Mesurement Set-up.

works pretty well in the angles close to $180^{\circ}$ degrees, while Time Filtering works better in the angles close to $90^{\circ}$. In fact INSIGHT corrects some of the effects of the foam basement, while the other methods (including the reference) cannot repair. In the case of the $90^{\circ}$ area, it is clear that time filtering works better since this is the worst case for the spatial or modal filtering (a plate orthogonal to the multiprobe).

Fig. 10 shows the results for the horizontal pattern. In this case, time filtering works better than the other two methods.

If the plate was placed in the side of the arch, time filtering had problems in the reconstruction. In this case, the direct ray and the reflected ray would get the receiver with equivalent delay for some of the probes. Also, that case is the best for the application of spatial filtering.

\section{CONCLUSIONS}

This paper has analyzed different post processing techniques to improve the results in antenna measurements. It is observed through measurements of a specific set-up that there is not a "best" algorithm for all the cases. Even for the same antenna, depending on the angular portion of the radiation pattern or the origin of the sources the results are improved in different ways.

Fortunately, these techniques can be applied simultaneously. Therefore, the combination of different techniques can improve significantly the results.

Unfortunately, time domain requires a multifrequency measurement, not always available. The required step and frequency band to have accurate results is under investigation in this moment.

\section{ACKNOWLEDGMENT}

The authors thank COST VISTA (Action IC1102) for the support of the stay of Dr. Javier García-Gasco in MVI during the measurements. Part of this work is supported by the SICOMORO project, TEC-2011-28789-C02-01, from the Spanish Government and SPADERADARCM Project S2013/ICE-3000, supported by Madrid Region Government.

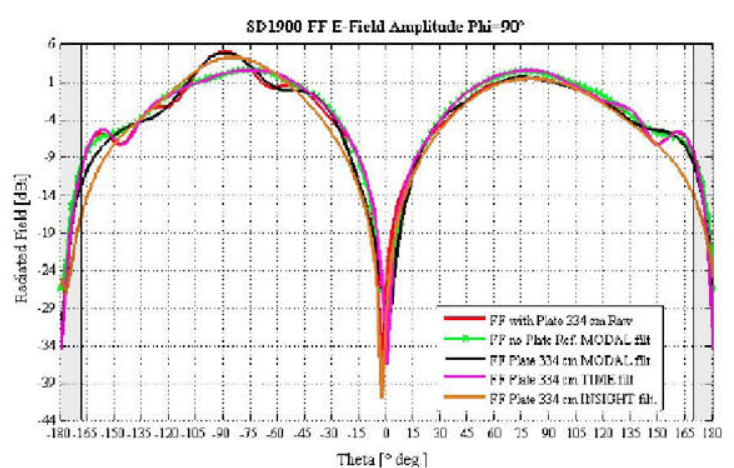

Fig. 9. Measurement results for the vertical plane..

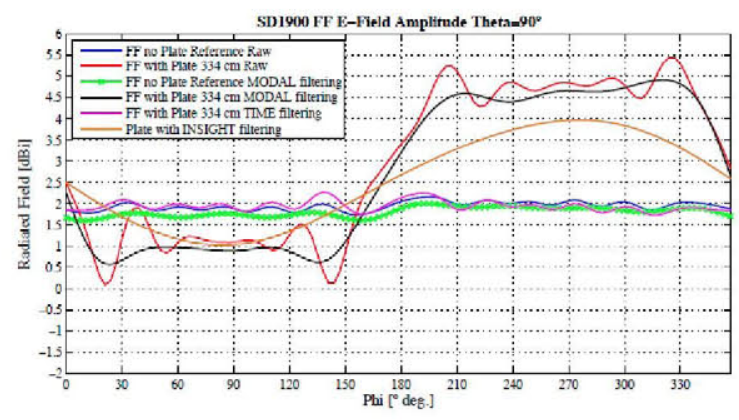

Fig. 10. Measurement results for the horizontal plane..

\section{REFERENCES}

[1] F. J. Cano-Fácila, S. Burgos, F. Martín, and M. Sierra-Castañer, New reflection suppression method in antenna measurement systems based on diagnostic techniques, IEEE Transaction on Antennas and Propagation, Vol. 59, no. 3, pp. 941-949, Mar. 2011.

[2] J. L. A. Quijano, L. Scialacqua, J. Zackrisson, L. J. Foged, M. Sabbadini, G. Vecchi "Suppression of undesired radiated fields based on equivalent currents reconstruction from measured data", IEEE Antenna and wireless propagation letters, vol. 10,2011.

[3] S. Gregson, A. Newell, and G. Hindman, "Reflection suppression in cylindrical near-field antenna measurement systems-cylindrical MARS," in Antenna Meaurement. Techniques Assoc., AMTA 2009, Salt Lake City, UT, November 1-6, pp. 119-125.

[4] D. Hess. "The IsoFilter ${ }^{\mathrm{TM}}$ Technique Isolating and Individual Radiator from Spherical Near-Field Data Measured in a Contaminated Environment". Proceedings of the European Conference on Antennas and Propagation 2007.

[5] MV-Echo Software. Microwave Vision Group. www.microwavevision.com.

[6] Aubin, J., Winebrand, M., Soerens, R., and Vinogradov, V., "Accurate near-fieldmeasurements using time-gating," Antenna Measurement Techniques Association Annual Symposium Proceedings, pp. 362-365, Nov. 2007.

[7] T. K. Sarkar and O. Pereira, "Using the matrix pencil method to estimate the parameters of a sum of complex exponentials," IEEE Antennas Propagat. Mag., vol. 37, No. 1, pp. 48-55, Feb., 1995. 